\section{A case of short-lasting unilateral neuralgiform headache with conjunctival injection and tearing (SUNCT). Discussion of clinical features and differential diagnosis}

\author{
M. Guidotti (西) • M. Mauri \\ Neurology Unit, \\ "Valduce" General Hospital, \\ via Dante 11, I-22100 Como, Italy \\ e-mail: mguidotti@valduce.it \\ Tel.: +39-031-324163 \\ Fax: +39-031-324165
}

Published online: 15 December 2005

\begin{abstract}
Chronic short-lasting headaches, in which trigeminal autonomic cephalalgias (TACs) are included, are relatively rare syndromes and not always well recognised. We present a case highly suggestive of short-lasting unilateral neuralgiform headache with conjunctival injection and tearing (SUNCT) and we try to affirm essential points to distinguish the diagnosis from other TACs and chronic short-lasting headaches. We conclude that the qualifying points for differential diagnosis are number and duration of attacks in a day, presence of autonomic features and lack of indomethacin effect.
\end{abstract}

Keywords SUNCT • TACs

\section{Case report}

A 47-year-old man was admitted to our Headache Centre for unilateral attacks of severe orbital headache, sometimes with temporal stabbing, extremely frequent (30-50 episodes in a day), lasting 15-30 s, associated with evident conjunctival injection and lacrimation, nasal congestion, rhinorrhoea and eyelid oedema. The pain quality was described as burning and throbbing. He was totally pain-free between attacks.

NMR of brain did not showed any pathologic condition.

The patient had presented this clinical condition for two weeks before arriving and he had experienced similar symptoms 1 and 4 years before, for about one month each time. In both events the clinical picture remitted spontaneously, and on these occasions the symptoms did not respond to therapy with subcutaneous sumatriptan, or to indomethacin.

No trigger zones were documented. Sometimes neck movements elicited the attacks.
The diagnosis we made was a short-lasting unilateral neuralgiform headache with conjunctival injection and tearing (SUNCT) case, with respect to diagnostic criteria of the revised IHS classification. Particularly, we think that the attacks' duration and frequency, the important autonomic concomitant features and the lack of indomethacin effect represented the qualifying points for diagnosis.

\section{Discussion}

SUNCT is one of the rarest trigeminal autonomic cephalalgias (TACs) and it shows a dramatic picture for the number of crises that create a condition of important disability. The worst characteristic is not the intensity of the crises, described as medium or severe, but never extremely severe like other chronic paroxystic cephalalgias, but the number of them and the intense autonomic involvement of the involved eye.

The syndrome was described first in 1978 [1] and afterwards characterised [2] for the extreme shortness of crises that last only a few seconds and the total absence of symptoms in the intercritical periods. The most intensive pain occurs in the orbital and peri-orbital regions and can irradiate ipsilaterally in the frontal, temporal and nasal zones, and to cheek and palate. The quality of pain is described as burning, stabbing or throbbing, and more rarely as an electric current. Paroxysms start and finish acutely, reaching the highest level in $2.3 \mathrm{~s}$. Single cases described the pain for longer times also, but the revised criteria of the International Headache Society indicate a duration between 10 and $120 \mathrm{~s}$ for SUNCT crises.

The medium number of crises is 28 in a day, with a range of 6-77, while the IHS indicates from 3 to 200 attacks in a day $[3,4]$.

In contrast with trigeminal neuralgia, there is no refractory period.

A great variety of symptoms are associated with the painful attacks. The most evident are conjunctival injection and lacrimation, which seem to mime cluster headaches. Ipsilateral nasal congestion, rhinorrhoea and eyelid oedema of the affected side are often seen at the same time. While trigger areas are not known, association with neck movements is sometimes seen before the SUNCT crises.

SUNCT enters in differential diagnosis with other TACs [1]. The most important aspect to consider is the relationship between duration and frequency of the attacks. Normally, when the duration of the attacks decreases, the frequency increases. Among the TACs, SUNCT and cluster headache are more common in men. 
Cluster headache and chronic paroxystic hemicrania are characterised by crises defined as "very severe", while SUNCT are "heavy and moderate".

The response to the therapy can be useful to identify the type of TAC too. Hemicrania continua and chronic paroxystic hemicrania respond dramatically to indomethacin, cluster headache to sumatriptan, and SUNCT does not have an affirmative response to either of these treatments.

Other useful aspects to differentiate the TACs are: association with local autonomic characteristics, the duration and the number of attacks, the daily/nocturnal phase of onset and possible trigger condition.

We can point out that SUNCT has an important association with conjunctival injection, more so than cluster headache and chronic paroxystic hemicrania, while hemicrania continua has less or no association and hypnic headache has none.

The very high number of crises is typical of SUNCT; higher than in cluster headache and chronic paroxystic hemicrania. The duration of a single attack is very short in SUNCT (a few seconds), and longer in the remaining TACs (from a few to various minutes).
There is no typical time of day for SUNCT attacks, while the other TACs occur during the night, with higher evidence for hypnic cephalalgia and cluster headache. SUNCT rarely has trigger zones, while occasionally neck movements induce the attacks.

On the basis of what we have reported, we conclude that the case we observed and described responds to the criteria of SUNCT diagnosis, not symptomatic.

\section{References}

1. Casucci G (2003) Chronic short-lasting headaches: clinical features and differential diagnosis. Neurol Sci 24:S101-S107

2. Goadsby PI, Lipton RB (1997) A review of paroxysmal hemicranias, SUNCT syndrome and other short lasting headaches with autonomic features, including new cases. Brain 120:193-209

3. Pareja JA, Shen JH, Kruszewski P, Caballero V, Pamo M, Sjaastad O (1996) SUNCT syndrome: duration, frequency, and temporal distribution of attacks. Headache 36:161-165

4. Pareja JA, Sjaastad O (1997) SUNCT syndrome: a clinical review. Headache 37:195-202 